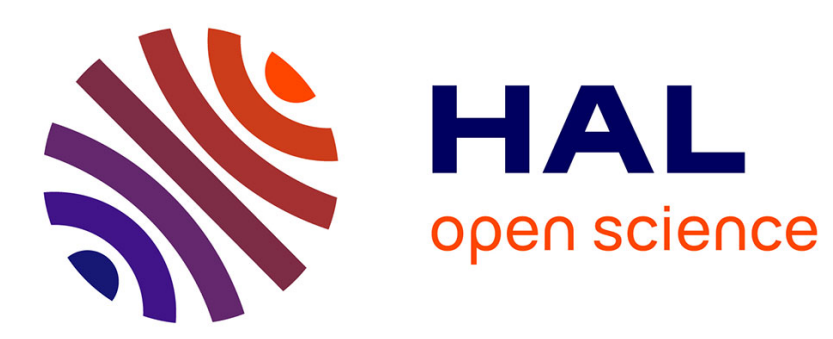

\title{
L'étude des phénomènes de surface dans les semi-conducteurs par la technique de l'effet de champ
}

R. Pick, M. Savelli

\section{To cite this version:}

R. Pick, M. Savelli. L'étude des phénomènes de surface dans les semi-conducteurs par la technique de l'effet de champ. J. Phys. Radium, 1960, 21 (10), pp.743-750. 10.1051/jphysrad:019600021010074300 . jpa-00236364

\section{HAL Id: jpa-00236364 https://hal.science/jpa-00236364}

Submitted on 1 Jan 1960

HAL is a multi-disciplinary open access archive for the deposit and dissemination of scientific research documents, whether they are published or not. The documents may come from teaching and research institutions in France or abroad, or from public or private research centers.
L'archive ouverte pluridisciplinaire HAL, est destinée au dépôt et à la diffusion de documents scientifiques de niveau recherche, publiés ou non, émanant des établissements d'enseignement et de recherche français ou étrangers, des laboratoires publics ou privés. 


\title{
EXPOSE ET MISE AU POINT BIBLIOGRAPHIQUE
}

\section{L'ÉTUDE DES PHÉNOMÈNES DE SURFACE DANS LES SEMI-CONDUCTEURS PAR LA TECHNIQUE DE L'EFFET DE CHAMP}

\author{
Par MM. R. PICK et M. SAVELLI, \\ Institut d'Études Nucléaires de l'Université d'Alger.
}

\begin{abstract}
Résumé. - Un certain nombre de techniques, dont l'effet de champ, permettent de rendre la densité des porteurs libres en surface différente de celle en volume, tout en respectant les conditions de micro-équilibre thermodynamique, ce qui rend valable l'utilisation de la théorie de la recombinaison de Shockley et Read. A partir de mesures de variation de conductivité et de décroissance de photoconductivité en fonction de l'effet de champ, il est possible de déterminer la densité des centres de recombinaison superficielle, leur niveau d'énergie et les probabilités de capture par ces centres d'un électron ou d'un trou.
\end{abstract}

Abstract. - A number of technical methods, among them the field effect, make the density of free carriers in the surface different from that in the volume, observing nevertheless the conditions of thermodynamic micro-equilibrium, this which gives valid the use of the theory of Shockley and Read recombination. From the measure of conductivity variations and photoconductivity decrease in relation to the field effect, it is possible to determine the density of centres of superficial recombination, their energy level and the capture probabilities of electrons or holes by these centres.

\section{INTRODUCTION.}

Depuis la mise en évidence de l'influence de l'état de surface sur le temps de recombinaison et les propriétés spectrales du bruit dans les semi-conducteurs [1], [2] ainsi que de l'effet channel dans les transistors [3], [4], [5], de nombreux chercheurs se sont intéressés à l'étude de la surface des semi-conducteurs.

On admet avec R. H. Kingston [6], depuis 1956, que l'effet de la surface s'explique par l'existence d'une couche d'oxyde : celle-ci introduit des niveaux supplémentaires d'impuretés, niveaux localisés soit dans la bande interdite, soit dans la bande de valence ou de conduction, aussi bien que dans la couche superficielle d'oxyde qu'à l'interface oxyde-semi-conducteur.

Au point de vue de la recombinaison, on admet [6], que les niveaux situés à l'interface oxyde-semi-conducteur ont, en général, une activité recombinative globale vis-à-vis des électrons et des trous, comparable à celle des centres de recombinaison en volume, et bien plus importante que celle des autres centres situés dans l'oxyde. Shockley et Read [7] ont développé dès 1953 une théorie de la recombinaison fondée sur le rôle de centres recombinatifs et de l'équilibre thermodynamique des porteurs libres entre ces centres, les bandes de valence et de conduction. On peut alors montrer que si, à un instant donné, on a injecté un excès $\Delta n$ d'électrons dans le semi-conducteur (com- pensés par $\Delta p$ charges positives réparties entre ces centres recombinatifs et la bande de valence, pour que le semi-conducteur reste électriquement neutre) les centres, en captant successivement un électron et un trou, annihilent, par la répétition cyclique de ce mécanisme, les paires électron-trou en excès, et le retour à l'équilibre se fait suivant une loi exponentielle, [8]

$$
\mathrm{d} \Delta n / \mathrm{d} t=-\Delta n / \tau
$$

où $\tau$ est appelé temps de recombinaison. Le nombre $U$ de recombinaisons par seconde est lié à $\tau$ par la relation :

$$
U=\Delta n / \tau
$$

$U$ est donné, d'après [7], par :

$U=N_{t} \frac{C_{n} C_{p}\left[\left(p_{0}+\Delta p\right)\left(n_{0}+\Delta n\right)-p_{0} n_{0}\right]}{C_{n}\left(n_{0}+\Delta n+n_{1}\right)+C_{p}\left(p_{0}+\Delta p+p_{1}\right)}$

dans laquelle : $N_{t}$ est la densité des centres de recombinaison de niveau d'énergie $E_{t} ; C_{n}$ représente la probabilité par seconde de capture d'un électron par un centre vide de niveau d'énergie $E_{t} ; C_{p}$ est la probabilité correspondante pour les trous; les quantités $p_{0}=n_{i} \mathrm{e}^{-\beta \varphi}$ et $n_{0}=n_{i} \mathrm{e}^{\beta \varphi}$ sont les densités des trous et des électrons à l'équilibre ; $n_{i}$ est une constante dépendant de la natura du semi-conducteur et de sa température $T ; \beta=1 / k T, k$ étant la constante de Boltzmann; $\varphi=F-E_{i}$, où $E_{\boldsymbol{i}}$ est l'énergie du milieu de la bande interdite et $F$ l'énergie du niveau 
de Fermi ; $p_{1}$ et $n_{1}$ sont les quantités équivalentes à $p_{0}$ et $n_{0}$ lorsque le niveau de Fermi a l'énergie $E_{t}$; $\Delta p$ et $\Delta n$ sont les densités supplémentaires des trous et des électrons dans les bandes correspondantes. Ces deux densités sont en général égales, aux seules conditions que $N_{t}, \Delta n$ et $\Delta p$ soient beaucoup plus petits que le plus grand des $n_{0}$ ou $p_{0}$.

Les équations (I-1) et (I-3), dont la démonstration est basée sur la notion de microéquilibre thermodynamique, sont valables localement, en tout point du cristal, pourvu que les électrons et les trous soient entre eux en équilibre thermodynamique ; en particulier, elles restent valables dans de nombreux cas où $n$ et $p$ ne sont pas uniformes dans l'ensemble du cristal : champ électrique local $\boldsymbol{E}$ compensé par des forces de diffusion, régime permanent, régime de diffusion lentement variable, etc...

Un certain nombre de techniques [6], dont l'effet de champ, permettent de rendre la densité des porteurs libres en surface différente de celle en volume, tout en respectant les conditions de microéquilibre, ce qui permet d'appliquer le modèle de Shockley et Read au phénomène de recombinaison en surface [9].

A partir de la mesure de $\tau$ et de la connaissance de certains paramètres macroscopiques liés au nombre de porteurs en surface, il est possible de déterminer $N_{t}$ ainsi que les constantes microscopiques $C_{n}, C_{p}$ et $E_{t}$ qui figurent dans (I-3).

Le chapitre II sera consacré à l'étude de l'effet de champ. Nous commencerons par montrer qualitativement que celui-ci entraîne une modification de la conductivité en surface, puis nous démontrerons les formules permettant de calculer cette variation de conductivité en fonction des seules valeurs de $\varphi$ : en volume soit $\varphi_{B}$ et à la surface soit $\varphi_{s}$. Dans le chapitre III, nous étudierons l'influence de $\varphi_{s}$ sur la recombinaison en surface et donnerons les formules permettant de la déterminer à partir de mesures de temps de recombinaison. Nous indiquerons, au chapitre IV, quelques-unes des difficultés expérimentales à résoudre et les mesures à réaliser afin de déterminer complètement $N_{t}$ et les constantes microscopiques $C_{n}, C_{p}$ et $E_{t}$.

\section{L'EFFET DE CHAMP.}

II-1. Description qualitative de l'effet de champ. L'effet d'un champ électrique intense appliqué perpendiculairement à la surface d'un semi-conducteur a été entièrement expliqué pour la première fois par G. C. E. Low [10].

L'expérience qu'il réalisait était la suivante : la surface d'une lame de semi-conducteur constituait l'une des faces d'un condensateur, dont l'autre était une lame métallique qui pouvait être portée à un potentiel $V$ (fig. 1). Low a constaté que, sous l'effet d'un potentiel, donc d'un champ électrique perpendiculaire à la surface, de la forme d'un échelon de Heaveside (figure 2-a), la résistance $R$ d'une plaquette de semi-conducteur avait une variation (voir figure $2-b$ ) brusque suivie de deux régimes exponentiels, de constantes de temps très différentes $\left[\tau_{1} \sim 100 \mu \mathrm{s} ; \tau_{2} \sim 1 \mathrm{~s}\right]$ dont le second tend à lui faire reprendre sa valeur initiale.

La cessation de champ s'accompagnait d'un phénomène identique au signe de la variation de résistance

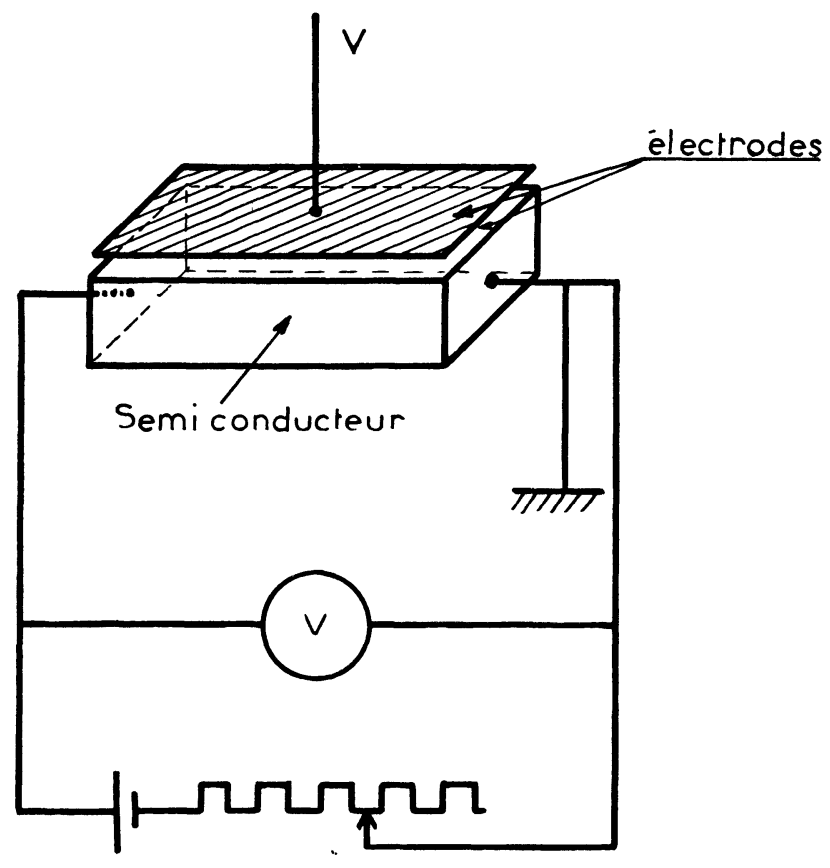

FIg. 1.

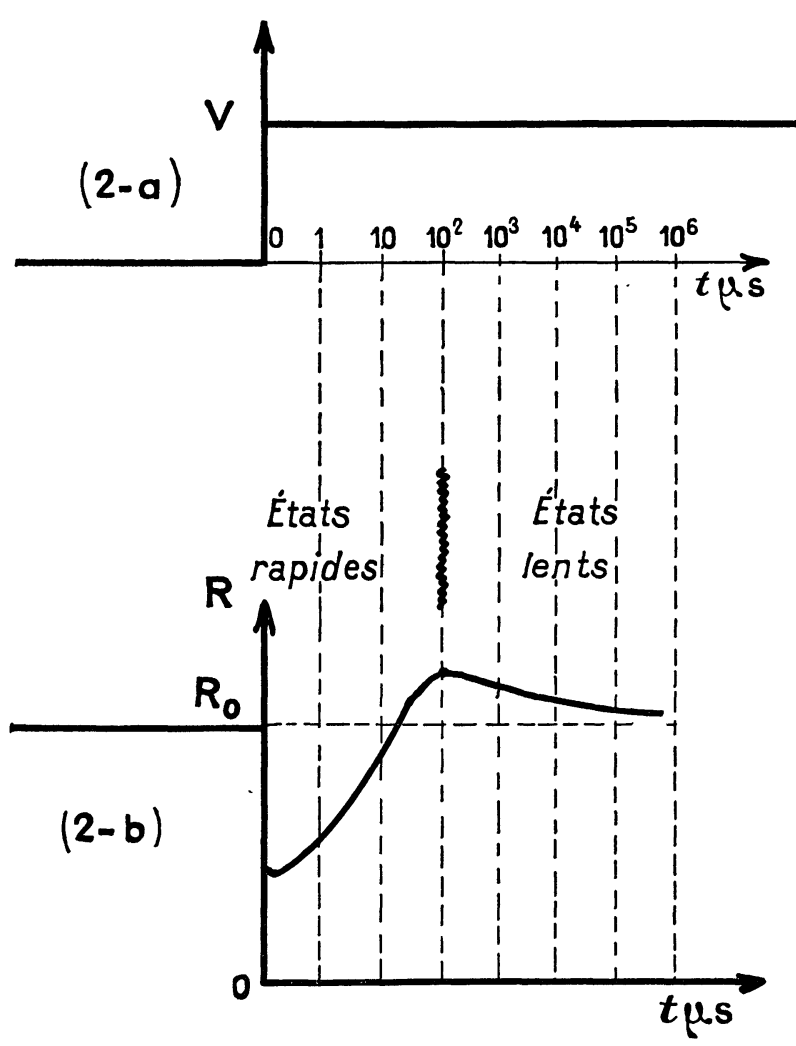

FIG. 2.

près. L'explication de ces variations de conductivité repose sur le fait suivant : la surface du semi-conducteur n'est pas suffisamment riche en états électroniques 
pour qu'il soit possible d'y placer les charges électroniques impliquées par l'existence du champ sans que cela n'entraîne une variation de la position de ces états vis-à-vis du niveau de Fermi.

Nous allons reprendre point par point l'expérience de G. C. E. Low en supposant, par exemple, le potentiel positif et le semi-conducteur de type $n$ ( $f g$. 3 ).

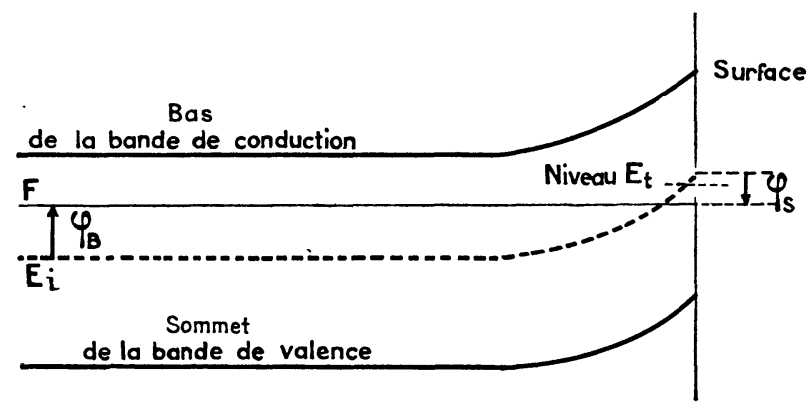

FIG. 3.

a) Lorsque le potentiel $V>0$ s'établit, les $Q_{s} / q=C V / q$ charges négatives qui vont se répartir à la surface du semi-conducteur sont fournies instantanément par le contact supposé ohmique de celui-ci avec la masse ( $q$ est la valeur absolue de la charge de l'électron). Ces charges restent susceptibles de se déplacer dans la bande de conduction, parallèlement à la surface ; il s'ensuit que la conductivité du cristal s'accroît brusquement.

Si, avant l'introduction de l'effet de champ, on avait:

$$
p_{s, 0}=n_{i} \mathrm{e}^{-\beta \varphi_{s, 0}} ; \quad n_{s, 0}=n_{\imath} \mathrm{e}^{\beta \varphi_{s, 0}}
$$

où $p_{s, 0}$ et $n_{s, 0}$ représentent les densités des trous et des électrons libres à la surface, on aura après l'application de $V$ :

$p_{s, a}=p_{s, 0}=n_{i} \mathrm{e}^{-\beta \varphi_{s, p}} \quad$ avec $\quad \varphi_{s, p}=\varphi_{s, 0}$

$n_{s, a}=n_{i} \mathrm{e}^{\beta \varphi s, n}$

avec $\varphi_{s, n}>\varphi_{s, 0}$

Remarquons que le nombre de charges introduites par unité de surface n'est pas égal à $n_{s, a}-n_{s, 0}$, mais à l'intégrale de cette quantité calculée sur un axe perpendiculaire à la surface et se dirigeant vers le volume du semi-conducteur. Mais comme $n_{s, a}-n_{\boldsymbol{s , 0}}$ garde un signe constant le long de cet axe, il s'ensuit que les variations de $n_{s, a}$ caractérisent bien l'ensemble des phénomènes au voisinage de la surface.

b) Par suite de la brusque augmentation du nombre d'électrons dans la bande de conduction, il n'y aura plus d'équilibre statistique entre ceux-ci, les trous de la bande de valence et les centres de recombinaisons. Il va donc y avoir recombinaison, ce qui, tout en gardant à $Q_{s}$ sa valeur aura deux effets :

D'une part, $n_{s, a}$ et $p_{s, a}$ vont décroître, ce qui diminuera la conductivité du semi-conducteur. D'après (II-2) et (II-3), nous voyons que $\varphi_{s, n}$ va diminuer et $\varphi_{s, p}$ croître et, à l'équilibre, nous aurons :

$$
\varphi_{s, n}(\text { équi })=\varphi_{s, p}(\text { équi })=\varphi_{s, e}
$$

où $\varphi_{s, e}$ est compris entre $\varphi_{s, 0}$ et $\varphi_{s, n}$ On en déduit

$$
\frac{1}{1+\mathrm{e}^{E_{t}-E_{i}-\varphi_{s, 0}}}<\frac{1}{1+\mathrm{e}^{E_{t}-E_{i}-\varphi_{s, e}}}<\frac{1}{1+\mathrm{e}^{E_{t}-E_{i}-\varphi_{s, n}}}
$$

D'où résulte que l'occupation des états électroniques de surface a augmenté ; un certain nombre des électrons supplémentaires resteront fixés sur les centres de recombinaisons. Le nombre de porteurs libres $n_{s, e}$ et $p_{s, e}$ à la surface est, à la fin de la recombinaison, différent de ce qu'il était en $a$ ) ainsi que de ce qu'il était avant l'application du champ.

c) A ce stade interviennent les niveaux localisés dans l'oxyde. Ces derniers peuvent offrir aux électrons un nombre d'états tellement important que la totalité des charges injectées par effet de champ peut s'y fixer sans que la position de leur niveau de Fermi en soit sensiblement modifié; seul, leur long temps de relaxation vis-à-vis $d u$ cristal les empêche de jouer un rôle pendant le court intervalle de temps nécessaire à la recombinaison décrite dans $b$ ). Cependant, très lentement, ils captent des électrons, ce qui a pour effet de déclencher un phénomène de générations de paires électron-trou ; au total, le nombre de porteurs libres sera redevenu au bout d'un temps très long ce qu'il était primitivement, soit $n_{\mathbf{s}_{\mathbf{0}}}$ et $p_{\mathbf{s , 0},}$. La conductivité a done repris sa valeur initiale.

II-2. Etude théorique de l'effet de champ. - Nous étudierons tout d'abord la variation du nombre de porteurs libres en surface introduite par le fait que $\varphi_{s}$ est différent de $\varphi_{B}$, en résolvant l'équation de Poisson correspondant au problème et nous en déduirons ensuite la variation correspondante de la conductivité [cf. fig. 3].

a) Théorie de L'efFet de champ statique (A L'ÉQUILIBRE) [11]. - Supposons $\alpha$ ) le semi-conducteur infini, limité par une face plane et soumis à des conditions identiques en chaque point.

ß) Les centres d'impuretés responsables des états de surface localisés dans une couche située à l'interface semi-conducteur-oxyde et d'épaisseur $e$ bien inférieure à la longueur de Debye, caractéristique de la courbure des bandes au voisinage de la surface, de telle sorte que l'effet de ces centres superficiels se transcrive simplement par la condition aux limites suivantes : $\varphi=\varphi_{s}$ à la surface $(x=0)$.

Soit $p$ la densité de charge locale, $V$ le potentiel local ; ]'équation de Poisson s'écrit, compte tenu de ce que le problème est unidimensionnel (hypothèse $\alpha$ ) et de ce que $\varphi=F-E_{i}=F+q V$

$$
\frac{\mathrm{d}^{2} V}{\mathrm{~d} x^{2}}=\frac{1}{q} \frac{\mathrm{d}^{2} \varphi}{\mathrm{d} x^{2}}=-\frac{\rho}{\varepsilon} \text {. }
$$

Les conditions aux limites sont :

$$
\left\{\begin{array}{l}
\varphi=\varphi_{S} \text { pour } x=0 \\
\varphi=\varphi_{B} \text { pour } x \rightarrow+\infty .
\end{array}\right.
$$

Admettons que l'ensemble des donneurs et accepteurs soit complètement ionisé en surface comme en volume, on a alors :

$$
\rho(x)=q\left[p(x)-n(x)+N_{D}-N_{A}\right]
$$

avec :

$$
n(x)=n_{i} \mathrm{e}^{\beta \varphi(x)} \quad \text { et } \quad p(x)=n_{i} \cdot \mathrm{e}^{-\beta \varphi(x)}
$$

$N_{D}$ et $N_{A}$ étant le nombre des donneurs et d'accepteurs par $\mathrm{cm}^{3}$. 
A l'intérieur du cristal, c'est-à-dire pour $x \rightarrow+\infty$, il n'y a pas de charge d'espace. Donc :

$0=q\left[p_{0}-n_{0}+N_{D}-N_{A}\right]$ avec $\left\{\begin{array}{l}n_{0}=n_{i} \mathrm{e}^{\beta \varphi_{B}} \\ p_{0}=n_{i} \mathrm{e}^{-\beta \varphi_{B}} .\end{array}\right.$

Et, par conséquent :

$$
\rho(x)=2 q n_{i}\left[\operatorname{sh} \beta \varphi_{B}-\operatorname{sh} \beta \varphi(x)\right]
$$

On peut donc écrire (II-2-1) sous la forme :

$$
\frac{\mathrm{d}^{2} \varphi}{\mathrm{d} x^{2}}=-\frac{2 q^{2} n_{i}}{\varepsilon}\left[\operatorname{sh} \beta \varphi_{B}-\operatorname{sh} \beta \varphi(x)\right] .
$$

La relation (II-2-4) s'intègre et donne, puisque $\mathrm{d} \varphi / \mathrm{d} x=0$ pour $\varphi=\varphi_{B}$ :

$\left[\frac{\mathrm{d} \varphi}{\mathrm{d} x}\right]^{2}=-\frac{4 q^{2} n_{i}}{\beta \varepsilon}\left[\beta\left(\varphi-\varphi_{B}\right) \operatorname{sh} \beta \varphi_{B}-\left(\operatorname{ch} \beta \varphi-\operatorname{ch} \beta \varphi_{B}\right)\right]$.

Soit :

$$
\begin{aligned}
& \mathrm{d} \varphi / \mathrm{d} x=\sqrt{K} F\left(\varphi, \varphi_{B}\right) \\
& \text { avec } K=4 q^{2} n_{i} / \beta \varepsilon \text { et } \\
& \begin{array}{l}
F\left(\varphi, \varphi_{B}\right) \\
=\lambda\left[-\beta\left(\varphi-\varphi_{B}\right) \operatorname{sh} \beta \varphi_{B}+\operatorname{ch} \beta \varphi-\operatorname{ch} \beta \varphi_{B}\right]^{1 / 2}
\end{array}
\end{aligned}
$$$$
\text { et avec } \lambda= \pm 1 \text { tel que } \lambda\left(\varphi-\varphi_{B}\right)<0 \text {. }
$$

Le nombre d'électrons en excès dans un cyclindre ayant sa base $\mathrm{d} y \mathrm{~d} z$ sur la surface et de hauteur infinie vers le semi-conducteur est :

avec

$$
\mathrm{d} y \mathrm{~d} z \int_{0}^{\infty}\left(n-n_{0}\right) \mathrm{d} x=\mathrm{d} y \mathrm{~d} z \Gamma_{n}
$$

$$
\Gamma_{n}\left(\varphi_{s}, \varphi_{B}\right)=\frac{n_{i}}{\sqrt{\bar{K}}} \int_{\varphi_{S}}^{\varphi_{B}} \frac{\mathrm{e}^{\beta \varphi}-\mathrm{e}^{\beta \varphi_{B}}}{F\left(\varphi, \varphi_{B}\right)} \mathrm{d} \varphi .
$$

On aura de même pour les trous :

$$
\Gamma_{p}\left(\varphi_{S}, \varphi_{B}\right)=\frac{n_{i}}{\sqrt{\bar{K}}} \int_{\varphi_{S}}^{\varphi_{B}} \frac{\mathrm{e}^{-\beta \varphi}-\mathrm{e}^{-\beta \varphi_{B}}}{F\left(\varphi, \varphi_{B}\right)} \mathrm{d} \varphi .
$$

Remarque $I$. - Il y a deux relations entre $\Gamma_{n}$ et $\Gamma_{p}$.

1) Si on applique le théorème de Gauss, à ce cylindre, on doit avoir :

$$
\frac{1}{q}\left(\frac{\mathrm{d} \varphi}{\mathrm{d} x}\right)_{S} \mathrm{~d} y \mathrm{~d} z=\frac{\mathrm{d} y \mathrm{~d} z}{\dot{z}} \int_{0}^{\infty} \rho(x) \mathrm{d} x .
$$

Soit en tenant compte de (II-2-6) :

$$
\frac{1}{q} \sqrt{K} F\left(\varphi_{S}, \varphi_{B}\right)=\frac{q}{\varepsilon}\left(\Gamma_{p}-\Gamma_{n}\right) .
$$

2) Si l'on fait une symétrie du schéma donnant l'allure des bandes au voisinage de la surface autour du milieu de la bande interdite en volume, on en dédui ${ }^{+}$ que $\Gamma_{p}\left(\varphi_{s}, \varphi_{B}\right)=\Gamma_{n}\left(-\varphi_{s},-\varphi_{B}\right)$.

Ce résultat peut être retrouvé algébriquement, en notant que $F\left(\varphi, \varphi_{B}\right)= \pm F\left(-\varphi,-\varphi_{B}\right)$, d'après la forme de $\Gamma_{n}$ et $\Gamma_{p}$.

Remarque II. - Si l'on suppose $\varphi \simeq \varphi_{B}$, un développement de l'équation (II-2-5) permet d'écrire :

$$
\left[\frac{\mathrm{d} \varphi}{\mathrm{d} x}\right]^{2}=\frac{8 q^{2} n_{i}}{\beta \varepsilon} \operatorname{ch} \beta \varphi_{B}\left[\operatorname{sh} \beta \frac{\varphi-\varphi_{B}}{2}\right]^{2} .
$$

On intègre, et par approximation au même ordre, il vient :

$$
\beta \frac{\varphi-\varphi_{B}}{4}=k \mathrm{e}-x / L_{D}
$$

avec :

$$
L_{D}=1 \sqrt{\frac{\varepsilon}{2 \beta n_{i} \operatorname{ch} \beta \varphi_{B}}}=\frac{1}{q} \sqrt{\frac{\varepsilon}{\beta\left(n_{0}+p_{0}\right)}} .
$$

Pour des conductibilités normales $\left(n_{0} \sim 10^{16} / \mathrm{cm}^{3}\right)$, $L_{D}$ qui peut être pris comme évaluation de la longueur d'onde de Debye, est de l'ordre de $10^{-5} \mathrm{~cm}$, de sorte que la variation de $\varphi$, de $\varphi_{s}$ à $\varphi_{B}$ ne doit pas dépasser le micron.

Remarque III. - Des calculs plus complets [12], [13] permettent de tenir compte de ce que les donneurs et les accepteurs peuvent n'être pas entièrement ionisés en surface.

b) ThÉorie SIMPlifiéE DE LA CONDUCTIVITÉ DE SURFACE. - Soit $G$ la conductivité d'une lame de semi-conducteur de longueur $l$, de section $A$ et de volume $V$, soit $V^{\prime}$ le volume du cristal en chaque point duquel on a $n=n_{0}$ et $p=p_{0}$ et $A^{\prime}$ la section correspondante de ce volume (voir fig. 4).

On a :

$$
G=\frac{1}{\int_{0}^{l} \frac{\mathrm{d} x}{\iint_{A} q\left(\mu_{\mathrm{n}_{0}} n_{0}+\mu_{\mathrm{p}_{0}} p_{0}\right) \mathrm{d} y \mathrm{~d} z+\iint_{A-A^{\prime}} q\left[\mu_{\mathrm{n}}\left(n-n_{0}\right)+\mu_{p}\left(p-p_{0}\right)\right] \mathrm{d} y \mathrm{~d} z}}
$$

Si $A-A^{\prime} \ll A$, ce qui est le cas, vu la valeur de $L_{D}$ devant les dimensions du cristal, il vient en posant :

$$
\begin{aligned}
& G=\sigma_{0} \frac{A}{l}+\quad \sigma_{0}=q\left(\mu_{n_{0}} n_{0}+\mu_{p_{0}} p_{0}\right) \\
& \frac{1}{l^{2}} \iiint_{v-v^{\prime}} q\left[\mu_{n}\left(n-n_{0}\right)+\mu_{p}\left(p-p_{0}\right)\right] \mathrm{d} x \mathrm{~d} y \mathrm{~d} z
\end{aligned}
$$

soit :

$$
G=G_{0}+\Delta G
$$

où $\Delta G$ est la variation de conductibilité due à l'effet de surface. Pour chercher à évaluer $\Delta G$, on peut faire l'une des deux hypothèses suivantes :
1) Les mobilités $\mu_{n}(x, y, z)$ et $\mu_{p}(x, y, z)$ en surface sont les mêmes qu'en volume. - Dans ce cas, on aura, compte tenu de la définition de $\Gamma_{n}$ et $\Gamma_{p}$ et en appelant $S$ la surface du cristal :

$$
\Delta G=\frac{1}{l^{2}} \iint_{S} q\left[\mu_{n_{0}} \Gamma_{n}+\mu_{p_{0}} \Gamma_{p}\right] \mathrm{d} y \mathrm{~d} z .
$$

2) La quantité $\left|\varphi_{s}-\varphi_{B}\right|$ est grande. - Schreiffer [14] a montré qu'au voisinage de la surface, la mobilité des porteurs parallèlement à cette surface était fortement diminuée, et ce d'autant plus que $\left|\varphi_{s}\right|$ et $\left|\varphi_{s}-\varphi_{B}\right|$ sont grands. Il définit la largeur $W$ de la région de surface fortement perturbée par :

$$
W=|k T / q E| \text { avec } q E=-(\partial \varphi / \partial x)_{s} .
$$


Il indique que lorsque $W$ est bien inférieur au libre parcours moyen $\lambda$ des porteurs libres dans le cristal, en première approximation, on avait :

$$
\Delta G=\frac{1}{l^{2}} \iint_{S} q\left[\frac{\mu_{n_{0}} \Gamma_{n}}{\lambda_{n_{0}}}+\frac{\mu_{p_{0}} \Gamma_{p}}{\lambda_{p_{0}}}\right] 3 W \mathrm{~d} y \mathrm{~d} z .
$$

Cela revient donc, en première approximation, à remplacer $\mu_{n}$ et $\mu_{p} \operatorname{par} 3 W \frac{\mu_{n_{0}}}{\lambda_{n_{0}}}$ et $3 W \frac{\mu_{p_{0}}}{\lambda_{p_{0}}}$.

c) Conséquences de L'Étude PRÉcédente. L'étude précédente montre qu'il est possible, connaissant les dimensions géométriques du cristal, et les quantités $\mu_{n_{0}}, \mu_{p_{0}}$ (et aussi $\lambda_{n_{0}}, \lambda_{p_{0}}$ dans le cas de l'approximation de Schreiffer) de calculer $\Delta G$ en fonction de $\varphi_{B}$ et $\varphi_{B}$. Pour $\varphi_{B}$ donné, la fonction $G\left(\varphi_{B}, \varphi_{B}\right)$ est approximativement parabolique avec un minimum pour $\varphi_{s} \simeq-\varphi_{B}$. En fait, par suite des différentes

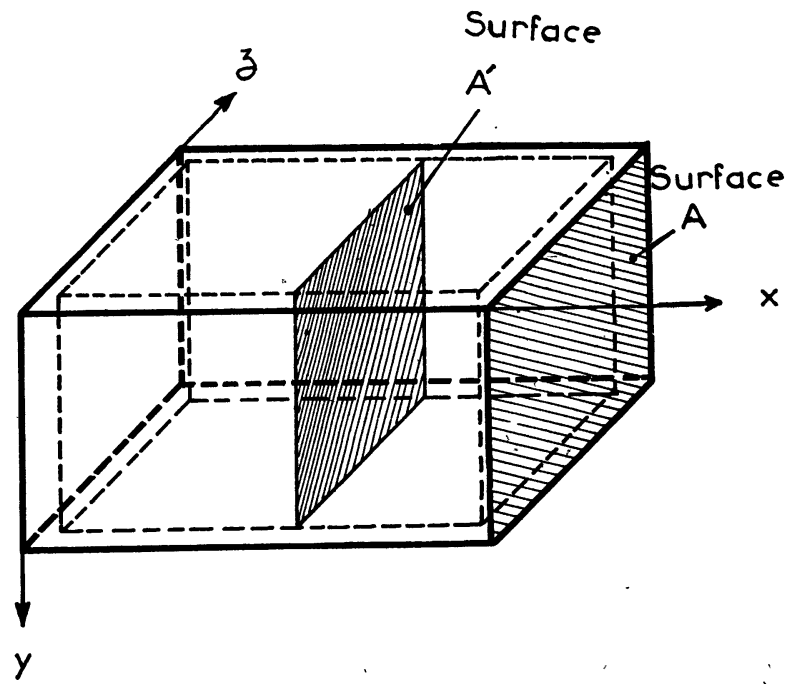

FIg. 34

impuretés adsorbées en surface, en l'absence de l'effet de champ, on a déjà $\varphi_{s} \neq \varphi_{B}$, donc $G=G_{0}+\Delta G_{0}$, où $\Delta G_{0}$, valeur de $\Delta G$ en l'absence de champ, est inconnue; il faut déterminer sa valeur pour relier l'accroissement $\left(\Delta G-\Delta G_{0}\right)$ de conductivité, due à l'effet de champ, à une variation de $\varphi_{s}$. Pour cela, [15] on réalise un champ de surface tel que la conductivité soit minimum soit $G_{\min }$ On sait alors que pour ce champ, $\varphi_{s} \simeq-\varphi_{B} ;$ pour toute autre valeur du champ, on mesure la différence $G-G_{\min }$, ce qui permet de connaitre la valeur de $\varphi_{s}+\varphi_{B}$ pour cette valeur du champ.

\section{ETUDE DE LA RECOMBINAISON SUPERFICIELLE.}

Nous définirons d'abord une vitesse de recombinaison en surface, analogue au temps de recombinaison défini pour le volume; nous donnerons son expression et étudierons sa variation en fonction de $\varphi_{s}$. Puis nous montrerons comment il est possible de déduire cette vitesse de recombinaison du temps de recombinaison effectif des porteurs libres, tout en tenant compte de la recombinaison en volume.
III-1. Formules de Shockley-Read pour les centres de surface. - Supposons qu'on injecte à l'intérieur d'un cristal un excès de trous et d'électrons. Au bout d'un temps très bref $\left(\sim 1^{-11} \mathrm{~s}\right)$, les ensembles constitués par les électrons d'une part, et par les trous d'autre part, se sont respectivement thermalisés, c'està-dire que l'occupation de chacune des bandes est régie par un niveau de Fèrmi $F_{n}$ pour les électrons et $\boldsymbol{F}_{\boldsymbol{p}}$ pour les trous, avec $F_{n} \neq F_{p}$.

Soient alors : $\Delta n_{s}$ l'excès d'électrons injectés situés en surface (en plus des $\left.n_{s}=n_{i} \mathrm{e}^{\beta \varphi_{s}}\right), \Delta p_{s}$ l'excès de trous correspondants (en plus des $p_{s}=n_{i} \mathrm{e}^{-\beta \varphi_{s}}$ ), et $N_{s}$ la densité superficielle des centres recombinatifs ; la formule de Shockley donne le nombre de recombinaisons par seconde et par unité de surface

$$
U=\frac{N_{s} C_{n} C_{p}\left[\left(n_{s}+\Delta n_{s}\right)\left(p_{s}+\Delta p_{s}\right)-n_{s} p_{s}\right]}{C_{n}\left(n_{s}+\Delta n_{s}+n_{1}\right)+C_{p}\left(p_{s}+\Delta p_{s}+p_{1}\right)} .
$$

Posons $F_{n}-E_{i}=\varphi_{n}$ et $F_{p}-E_{i}=\varphi_{p}$; on peut alors écrire :

$$
\left.\begin{array}{l}
n_{s}+\Delta n_{s}=n_{i} \mathrm{e}^{\beta \varphi_{n}} \\
p_{s}+\Delta p_{s}=n_{i} \mathrm{e}^{-\beta \varphi p}
\end{array}\right\}
$$

Le produit $\left(n_{s}+\Delta n_{s}\right) \cdot\left(p_{s}+\Delta p_{s}\right)$ est égal à $n_{i}^{2} \mathrm{e}^{\beta\left(\varphi_{n}-\varphi_{p}\right)}=n_{i}^{2} \mathrm{e}^{\beta\left(F_{n}-F_{p}\right)}$, il ne dépend pas de $E_{i}$, et

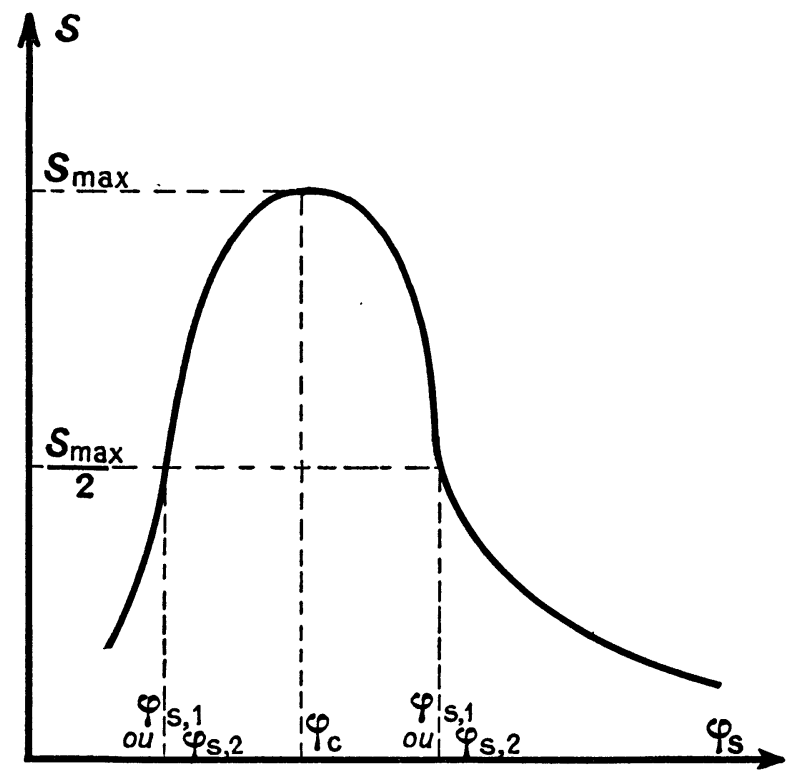

Fig. 5 .

est égal à $\left(n_{0}+\Delta n_{0}\right) \times\left(p_{0}+\Delta p_{0}\right)$. Le facteur entre crochets du numérateur de (III-1) est donc égal au facteur correspondant de (I-3). Négligeons $\Delta n_{s}$ et $\Delta p_{s}$ au dénominateur de (III-1), et supposons une injection faible de telle sorte que $\Delta n_{0}=\Delta p_{0}$, on tire alors de (III-1)

$$
s=\frac{U}{\Delta n_{0}}=\frac{N_{s}\left(p_{0}+n_{0}\right) C_{n} C_{p}}{C_{n}\left(n_{s}+n_{1}\right)+C_{p}\left(p_{s}+p_{1}\right)} .
$$

Posons : $C_{p} / C_{n}=\mathrm{e}^{\varepsilon \beta \varphi_{c}}$, la relation (III-3) devient [16] :

$$
s=N_{s} \sqrt{C_{n} C_{p}} \frac{\operatorname{ch} \beta \varphi_{B}}{\operatorname{ch} \beta\left(\varphi_{c}-\varphi_{s}\right)+\operatorname{ch} \beta\left(\varphi_{c}-E_{t}+E_{i}\right.}
$$


La quantité $\left(E_{t}-E_{i}\right)$ ne dépend que de l'énergie des niveaux des centres recombinatifs, donc est indépendante de $\varphi_{s}$; par suite, la dépendance de $s$ en fonction de $\varphi_{s}$ est simple. On trouve (voir fig. 5) en supposant $\operatorname{ch} \beta\left(\varphi_{c}-E_{t}+E_{i}\right) \gg 1$ :

a) que $s$ est maximum pour $\varphi_{s}=\varphi_{c}$,

b) que pour $s=\frac{1}{2} s_{\max }$ on a :

soit :

$$
\left|\varphi_{s}-\varphi_{c}\right|=\left|\varphi_{c}-E_{l}+E_{i}\right|
$$

$$
\varphi_{s, 1}=E_{t}-E_{i} \quad \text { et } \quad \varphi_{s, 2}=2 \varphi_{c}-\left(E_{t}-E_{i}\right)
$$

$\varphi_{c}$ dépendant de la température, une seule des deux valeurs de $\varphi_{s_{.1}}$ ou $\varphi_{s_{2},}$ n'en dépendra pas. Par conséquent, il est en principe possible, si l'on possède les valeurs de $s$ en fonction de $\varphi_{s}$, d'une part de déterminer $\varphi_{c}$ donc $\frac{C_{p}}{C_{n}}$, et d'autre part, en réalisant les expériences à diverses températures, de déterminer laquelle des deux valeurs de $\varphi_{s}$ pour $\frac{1}{2} s_{\max }$ est indépendante de $T$, donc de déterminer $\left(E_{t}-E_{i}\right)$.

Une étude plus poussée de cette question a été réalisée par Dousmanis [17].

III-2. Mesure de la vitesse de recombinaison en surface par la vitesse de la décroissance de la conductivité. - Nous allons étudier la décroissance de la conductivité pour un échantillon de forme parallélépipédique, de faible épaisseur, forme utilisée lorsque I'on veut réaliser simultanément sur cet échantillon un effet de champ et une étude de la recombinaison superficielle. Par injection électrique ou optique, on introduit des porteurs supplémentaires (par exemple des trous dans un cristal de type $n$ ). La longueur du cristal est $L$, sa section a pour côtés $2 A, 2 B$ avec $A \sim 10^{-2} \mathrm{~cm}<B$.

a) Recombinaison purement superficielle. Supposons que les centres de recombinaison n'existent qu'en surface. Dans une tranche d'épaisseur $\mathrm{d} x$, il y a $4 A B \Delta p \mathrm{~d} x$ trous supplémentaires et d'après (III'-3) il s'en recombine $4(A+B) \Delta p s \mathrm{~d} x$ durant l'unité de temps. Donc :

$$
4 A \cdot B \cdot \frac{\mathrm{d} \Delta p}{\mathrm{~d} t}=-4(A+B) s \Delta p .
$$

Ceci donne une décroissance exponentielle pour $\Delta p$, de constante de temps $\tau$ telle que

$$
\frac{1}{\tau}=s\left(\frac{1}{A}+\frac{1}{B}\right) .
$$

Remarque: La formule (III-5) montre que $s$ a la dimension d'une vitesse. C'est ce qu'on appelle la vitesse de recombinaison superficielle.

b) Influence de la recombinaison en volume. Supposons qu'il existe aussi des centres de recombinaison en volume auxquels soient associés la constante $\tau_{B}$. On peut alors écrire en sommant les recombinaisons en volume (I-1) et en surface (III-5)

$$
\frac{\mathrm{d} \Delta p}{\mathrm{~d} t}=-s\left(\frac{1}{A}+\frac{1}{B}\right) \Delta p-\frac{\Delta p}{\tau_{B}}
$$

ce qui donne une décroissance exponentielle, de constante telle que :

$$
\frac{1}{\tau}=\frac{1}{\tau_{B}}+s\left(\frac{1}{A}+\frac{1}{B}\right)
$$

Remarque : Dans la formule (III-3), $U$ représente le flux de porteurs minoritaires arrivant à la surface et s'y recombinant par seconde, et par unité de surface, et $q U$ le courant de porteurs minoritaires arrivant à la surface. Ce courant étant causé par les seules forces de diffusion, si $D$ est la constante de diffusion ambipolaire, on a done :

$$
s \Delta p=-D(\mathrm{~d} \Delta p / \mathrm{d} n) .
$$

La dérivée $\mathrm{d} / \mathrm{d} n$ étant prise normalement à l'unité de surface, et orientée vers l'intérieur.

On peut alors écrire que, par unité de volume, la variation dans le temps de $\Delta p(x, y, z)$ est due à la recombinaison locale en volume, et à l'influence des forces de diffusion.

$$
\frac{\mathrm{d} \Delta p}{\mathrm{~d} t}=-\frac{\Delta p}{\tau_{B}}+D \Delta(\Delta p)
$$

La condition aux limites sur la surface étant donné par (III-9) ; la résolution de (III-10) par la méthode des fonctions propres, [18], donne pour $t \rightarrow \infty$ une décroissance exponentielle de constante de temps $\tau$ telle que, [19] :

$$
\frac{1}{\tau}=\frac{1}{\tau_{B}}+D\left(\alpha^{2}+\beta^{2}\right)
$$

$\alpha$ et $\beta$ étant les plus petites solutions en valeur absolue des équations suivantes :

$$
\left.\begin{array}{l}
\alpha A \operatorname{tg} \alpha A=s A \mid D \\
\beta B \operatorname{tg} \beta B=s B \mid D .
\end{array}\right\}
$$

Il s'ensuit que l'on aura :

$$
\begin{aligned}
& \frac{1}{\tau}=\frac{1}{\tau_{B}}+s\left(\frac{1}{A}+\frac{1}{B}\right) \quad \text { si } s \ll \frac{3 D}{2}\left(\frac{1}{A}+\frac{1}{B}\right) \\
& \frac{1}{\tau}=\frac{1}{\tau_{B}}+\frac{\pi^{2} D}{4}\left(\frac{1}{A^{2}}+\frac{1}{B^{2}}\right) \quad \text { si } s \gg \frac{3 D}{2}\left(\frac{1}{A}+\frac{1}{B}\right) \text {. }
\end{aligned}
$$

Donc la mesure de décroissance de la conductivité d'un cristal après injection de porteurs supplémentaires permettrà, si l'on connait $\tau_{B}$, de déterminer $s$, dans le cas où $s \ll \frac{3 D}{2}\left(\frac{1}{A}+\frac{1}{B}\right)$.

\section{MÉTHODES EXPÉRIMENTALES DE L'EFFET DE CHAMP ET EXPLOITATION.}

Nous allons indiquer ici, brièvement, d'une part le montage du semi-conducteur soumis à l'effet de champ et, d'autre part, les différentes mesures à réaliser que nous récapitulerons ensuite dans le tableau I.

a) Teghnique Du montage. - Les deux surfaces, bien polies, d'une fine lame de germanium forment les électrodes d'un double condensateur (voir fig. 6) rempli de diélectrique [mica $\varepsilon=10 \varepsilon_{0}$ [16], [20], titanate de baryum $\varepsilon=300 \varepsilon_{0}$ [21]], les contre-électrodes étant réunies entre elles et portées au potentiel $V$ permettant l'injection des porteurs à la surface du germanium. Il est nécessaire d'éviter au maximum les inhomo- 
généités de la surface des électrodes et du diélectrique pour avoir le champ le plus uniforme possible à la surface du germanium. De la sorte, la valeur de $\varphi_{s}$

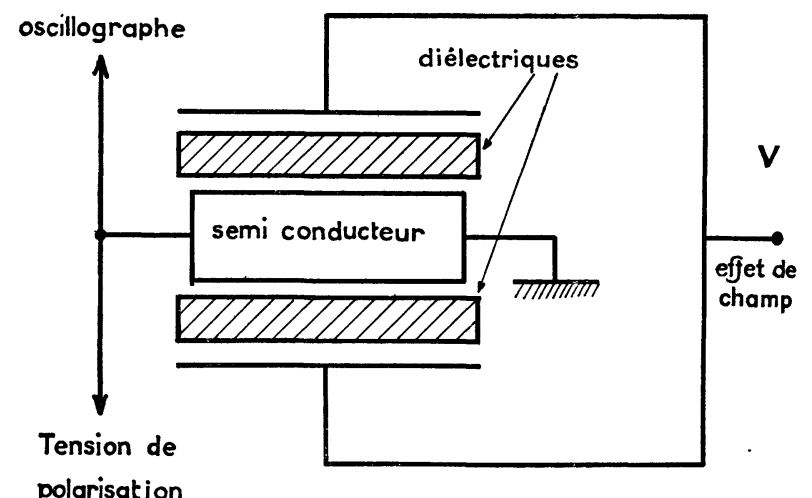

FIG. 6.

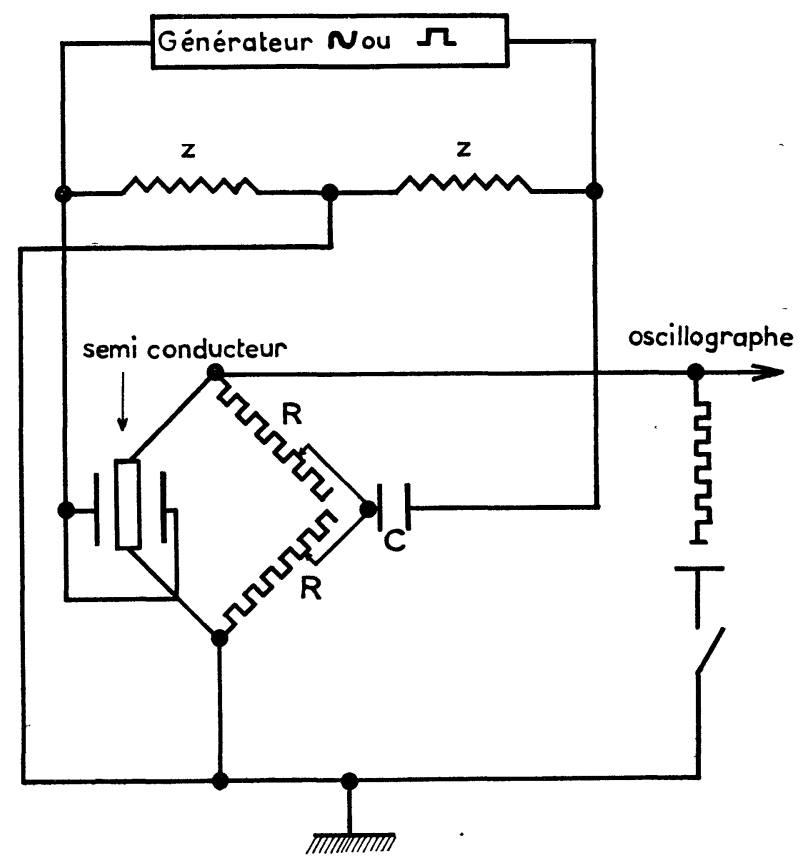

FIG. 7.

est à peu près la même sur toute l'étendue de la surface du cristal, ce qui permettra un bon accord entre les courbes $S=S\left[\varphi_{s}\right]$ expérimentales et théoriques (formule III-3).

b) Mesure de la variation de la conductibilité EN FONCTION DE L'EFFET DE CHAMP. - Nous avons vU en II-1 que les états lents tendaient à neutraliser l'effet de champ. Il faut faire la mesure de $\Delta G$ tout de suite après l'établissement du champ et en un temps petit devant la constante de temps ( $\sim 1$ sec) de ces états lents. Ceci conduit à faire une mesure de $\Delta G$ en appliquant une tension $V$ périodique $(\nu \sim 50 \mathrm{c} / \mathrm{s})$ et symétrique par rapport à $V=0$. De cette façon, le champ est, en moyenne, nul, et il n'y a pas de charges électriques qui finissent par s'accumuler dans les états lents. Le potentiel $V$ est, en général, sinusoïdal ou en crénaux. Pour éviter qu'au signal de conductivité vienne se superposer la dérivée de la tension $V$, élaborée par la constante de temps propre de l'ensemble condensateur plus germanium, on peut utiliser un montage symétrique du type Zener [22] que l'on équilibre en l'absence de courant de polarisation (voir fig. 7).

La mesure de $\Delta G$ en fonction de $V$ se fera à l'oscillographe après une amplification en courant continu. En comparant le $\Delta G=\Delta G(V)$ expérimental à la courbe théorique $\Delta G=\Delta G\left(\varphi_{s}\right)$ (voir formule II-3-4) obtenue pour les valeurs $A, B, L, \mu_{n_{0}}, \mu_{p_{0}}$ et $\varphi_{B}$ du cristal, on déduit la correspondance $V \stackrel{=}{=}\left(\varphi_{s}\right)$.

c) Mesure de la décroissance de la conducTIVITÉ. - Ceci est une mesure classique, que l'injection des porteurs ait lieu par voie optique ou électrique. Cette injection est réalisée pendant que l'échantillon est simultanément soumis au potentiel $V$. De la fonction mesurée $\tau=\tau(V)$ on déduit par (III-8) la courbe $s=s(V)$; donc compte tenu de $b$ ) la fonction $s=s\left(\varphi_{s}\right) ;$ par suite on détermine $\varphi_{c}$, donc $\frac{C_{n}}{C_{p}}$, et les valeurs de $\varphi_{s, 1}$ et $\varphi_{s, 2}$, l'une d'entre elles devant être $E_{t}-E_{i}$.

d) Détermination de $N_{s}$ Par la mesure de la CAPAGITÉ $C$ Du CONDENSATEuR. - On mesure la capacité $C \mathrm{du}$ condensateur ainsi formé, d'où l'on déduit la charge $Q_{s}=Q_{s}\left(\varphi_{s}\right)=C V\left(\varphi_{s}\right)$ introduite dans le semi-conducteur. On calcule théoriquement la charge d'espace $Q_{C E}=Q_{C E}\left(\varphi_{s}, \varphi_{B}\right)=S q\left(\Gamma_{n}-\Gamma_{p}\right)$ et l'on déduit la charge $Q_{E S}$ effectivement placée dans les états de surface, soit :

D'autre part l'on a :

$$
Q_{E S}=\sum_{k} q S N_{s, k} \frac{1}{1+\mathrm{e}^{\left(E t_{k}-E i-\varphi s\right) \beta}} .
$$

S'il n'y a qu'un niveau $E_{t}$, l'on déduit $N_{s}$, donc d'après (III-4) la quantité $C_{n} C_{p}$.

Remarque: Si l'accord est mauvais entre $Q_{E S}(\varphi z)$ mesurée et théorique, l'on doit chercher à l'améliorer en supposant deux niveaux $E_{t_{1}}$ et $E_{t_{2}}$ pour les états de surface et en déduire $N_{s_{1}}$ et $N_{s_{2}}$.

Sur le tableau I nous avons dressé un schéma de l'ensemble des opérations théoriques et expérimentales à effectuer dans une étude complète de l'effet de champ.

\section{CONCLUSION.}

Nous avons montré dans ces différents chapitres, en quoi consistait l'effet de champ, et comment on le mettait en œuvre pour déterminer les propriétés de surface des semi-conducteurs. Cette technique possède suffisamment d'avantages pour qu'elle ait été utilisée concurremment avec d'autres (Cycle de BrattainBardeen [22]. - Mesure de potentiel de contact [23], [24] ou de résistivité, avec et sans illumination permanente de l'échantillon) pour déterminer les caractéristiques des niveaux de surface, ainsi que pour préciser leur origine exacte.

Elle présente, en revanche, d'assez importantes difficultés de réalisation dues à la nécessité d'obtenir des cristaux qui restent en surface monocristallins, tout 


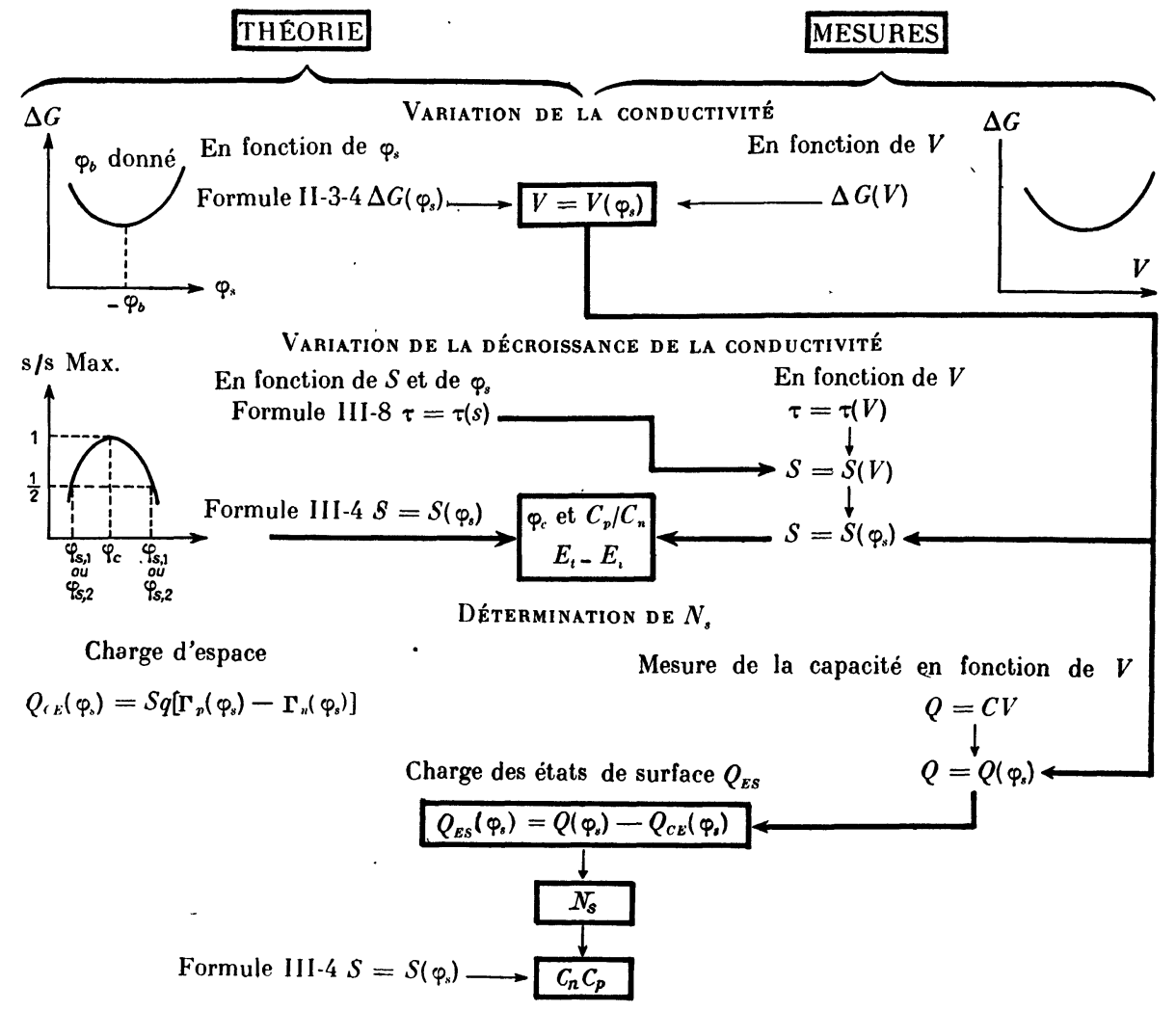

en étant assez bien polis pour que l'ensemble de la surface soit soumis à un effet du champ homogène. L'absence de précautions suffisantes explique sans doute certains désaccords expérimentaux.

D'un autre côté, l'étude des états de surface présente toujours une même difficulté tenant à l'extrême sensibilité des surfaces aux atmosphères extérieures. Pour tenter de clarifier le problème Many [25] et surtout Rhzanov [20], [26] et son école [27] ont travaillé sous des vides de plus en plus poussés. Si ces études ont apporté des résultats nouveaux sur le caractère de certains états lents, la dispersion concernant les résultats sur les états rapides a, au contraire, augmenté [28]. Il est, à l'heure actuelle, assez clair que, non seulement la densité des états recombinatifs, mais aussi leur énergie varie avec la quantité et la nature du gaz adsorbé par la surface.

Manuscrit reçu le 18 mars 1960.

\section{BIBLIOGRAPHIE}

[1] Maple (T. G.), Bess (L.) et Gebbie (H. A.), J. Appl. Physics, 1955, 26, 490.

[2] Montgomery (H. C.), Bell Syst. Techn. J., 31, 950.

[3] Kingston (R. H.), Phys. Rev., 1955, 98, 1767.

[4] Kingston (R. H.) et MacWorthe r (A. L.), Phys. Rev., 1956, 103, 534 .

[5] Statz (H.), De Mars (G.) et al., Phys. Rev., 1957, 106,455 .

[6] Kingston (R. H.), J. Appl. Physics, 1956, 27, 101

[7] Shockley (W.) et Read (W. T.), Phys. Rev., 1952, $87,835$.

[8] Sa Ndiford (D. J.), Phys. Rev., 1957, 105, 524.

[9] Stevenson (D. T.) et Keyes (R. S.), Physica, 1954, 20,1041 .

[10] Low (G. G. E.), Proc. Phys. Soc., 1955, 68 B, 10.

[11] Kingston (R. H.) et Neustader (S. F.), J. Appl. Physics, 1955, 26, 718.

[12] Dousmanis (G. C.) et Duncan (R. C.), J. Appl. Physics, 1958, 29, 1627.

[13] Seiwartz (R.) et Green (M.), J. Appl. Physics, 1958, 29, 1034 .

[14] Schreiffer (J. R.), Phys. Rev., 1955, 97, 641

[15] Brown (W. L.), Phys. Rev., 1955, 100, 590.
[16] Many (A.), Harnick (E.) et Margoninsky (Y.), Semiconductor Surface Physics. University Press of Pensylvania, p. 85.

[18] Stevenson (D. T.) et Keyes (R. S.), J. Appl. Physics, 1955, 26, 190.

[19] SHOcKLEY (W.), Electrons and Holes in semiconductors. D. Van Nostrand et Gie, p. 319.

[20] Rhzanov (A. V.) et al., Soviet Physics (Technical Physics), 1958, 28, 2419.

[21] Montgomery (H. C.) et Brown (W. L.), Phys. Res., 1956, 103, 865 .

[22] Garett (G. C. B.) et Brattain (W. H.), Bell. Syst. Tech. J., 1956, 35, 1019 et 1041 .

[23] BardeEN (J.), Coovert (R.), Morrison (S.), Schreiffer (J.) et Son (R.), Phys. Rev., 1956, 104, 47.

[24] Wang (S.) et Wallis (G.), Phys, Rev, 1957, 105, 1459

[25] Many (A.) et Gerlich (D.), Phys. Rev., 1957, 107, 404.

[26] Rhzanov (A. V.) et al., Soviets Physics (Technical Physics), 1957, 27, 2274.

[27] Lyashenko (V. I.) et Chernaya (N. S.), Soviets Physics (Solid State), 1959, 1, 799.

[28] Many (A.) et al., Phys. Rev., 1955, 101, 1433. 